\title{
BMJ Health \& Care Informatics \\ Digital health and care in pandemic times: impact of COVID-19
}

\author{
Niels Peek, ${ }^{1,2}$ Mark Sujan,, ${ }^{3,4}$ Philip Scott (D) ${ }^{5}$
}

To cite: Peek N, Sujan M, Scott P. Digital health and care in pandemic times: impact of COVID-19. BMJ Health Care Inform 2020;27:e100166. doi:10.1136/ bmjhci-2020-100166

Received 04 May 2020 Accepted 02 June 2020

Check for updates

(c) Author(s) (or their employer(s)) 2020. Re-use permitted under CC BY-NC. No commercial re-use. See rights and permissions. Published by BMJ.

${ }^{1}$ Division of Informatics, Imaging and Data Science, School of Health Sciences, School of Health Sciences, University of Manchester, Manchester, UK ${ }^{2} \mathrm{NIHR}$ Manchester Biomedical Research Centre, The University of Manchester, Manchester, UK

${ }^{3}$ Warwick Medical School, University of Warwick, Coventry, UK

${ }^{4}$ Human Factors Everywhere Ltd, Woking, UK

${ }^{5}$ School of Computing, University of Portsmouth, Portsmouth, UK

Correspondence to

Prof Niels Peek;

niels.peek@manchester.ac.uk

\section{INTRODUCTION}

The COVID-19 pandemic has created many challenges for health and care services worldwide and has led to one of the largest societal crises in last century. It has also been a test for the maturity of digital health technologies, be it for frontline care, surveillance or discovery of new strategies. In this editorial, we reflect on developments in service delivery, artificial intelligence (AI) and data sharing instilled by the COVID-19 crisis and consider which conclusions can be drawn so far.

\section{SERVICE DELIVERY}

Primary care and outpatient hospital care have long held the promise that they could largely be delivered digitally, but until very recently, the scale of digital transformation has been modest. Aspirations around digital transformation had to be tempered by the reality of system inertia and slow speeds of adoption due to a multitude of barriers related to reimbursement, accreditation and human factors. For instance, in the USA, only $20 \%$ of states require payment parity between telemedicine and in-person services. ${ }^{1}$

The COVID-19 pandemic has dramatically changed all this in a matter of weeks. The potential of digital health technologies to protect patients, clinicians and the community from exposure has been broadly recognised and catalysed the uptake of these technologies in a way not hitherto experienced. ${ }^{2}$ Many countries have adopted digital-first strategies, remote monitoring and telehealth platforms to enable healthcare provision without physical interactions. In the UK, primary care has embraced telehealth at scale and deployed a new digital-first pathway as a route to managing streaming of care to the appropriate place. ${ }^{3}$ This would have been beyond the limits of the possible only a few months ago.

This rapid change was possible for three reasons. First, many companies could offer solutions by adapting software that already existed, rather than starting from scratch.
The technology was sufficiently mature to be deployed at scale when COVID-19 struck. Second, many countries have relaxed privacy and data protection regulations for video and other communications technologies during the crisis ${ }^{4}$; the General Data Protection Regulations, which apply in the UK and the European Union, already include a clause excepting work in the overwhelming public interest. Third, change was necessary because governments required that any care that does not require physical interaction must now be provided through remote consultation. ${ }^{5}$

Remote management is possible for many patients that are seen in primary care and hospital outpatient clinics. This includes patients with COVID-19 that can be managed remotely with advice on symptomatic management and self-isolation. ${ }^{6}$ Moreover, this type of management can still be delivered by healthcare workers that are quarantined after infection or exposure. Telehealth tools have also been suggested as a form of electronic personal protective equipment (PPE) that can be used by acute care clinicians to evaluate hospitalised patients while avoiding physical proximity. ${ }^{7}$ Indeed, telehealth has already been described as a 'virtually perfect solution' for COVID-19.

The key question is whether healthcare services would, and should, return to predominantly face-to-face interactions after the COVID-19 pandemic. Former barriers to digital transformation may return when temporary provisions for COVID-19 shift out of force. Some clinicians have already said that they would prefer to continue remote consultations where possible, but others have highlighted the need for larger structural change to avoid exacerbation of health inequalities. ${ }^{9}$ Some remote digital technologies, such as digitalfirst primary care, are under-researched, and there exist serious concerns regarding their safety. ${ }^{10}$ Much more high-quality research into these technologies is needed to enable our societies making well-informed decisions for the future. 


\section{ARTIFICIAL INTELLIGENCE}

Even before the COVID-19 pandemic hit health systems worldwide, hopes were high that the widespread development and deployment of AI within healthcare could help overstretched care providers through the development of new drugs, the optimisation of data and information flows and the personalised and timely delivery of care. ${ }^{11}$ With the pandemic in full swing, it is timely to reflect on how AI can help (or has helped) health systems to manage the crisis and to consider the role of AI as countries prepare for a potential second wave of infections linked to coronavirus.

At the outset of the current crisis, innovative AI-based analysis of social media data and news reports helped to predict the spread of the outbreak. Canadian company Blue Dot is credited with being first to recognise an unusual cluster of pneumonia cases in Wuhan before official sources confirmed this as COVID-19. Large amounts of data can be gathered and aggregated quickly from a range of sources, such as Twitter, Facebook, local news outlets and public health statistics to reconstruct and then potentially predict the spread and the behaviour of the COVID-19 outbreak. These early successes at modelling and predicting disease behaviour are encouraging, but questions need to be asked about the reliability and quality of the data that go into the AI.

Social media analysis could potentially be triangulated further with mobile phone data that capture people's movements to give a real-time prediction of risk and disease spread. Such tracing of movement could support the public with complying more easily with social distancing by being routed away from crowded areas. Apple and Google have formed a partnership to develop an app to support contact tracing. This app takes a decentralised approach, where data are stored locally on each person's phone. In the UK, NHSX has rejected this partnership's design and opted for the development of a proprietary app where data will be held centrally on NHS servers. ${ }^{12}$ This raises ethical and privacy concerns, in particular, around the potential for data sharing beyond the immediate COVID-19 pandemic. There are also uncertainties about the actual utility of contact tracing due to the lack of adequate, validated risk models and due to the need to ensure widespread use of the app within the population.

Babylon Health, already a controversial player in the AI healthcare market prior to COVID-19, extended its symptom-checking app with a specific COVID-19 decision algorithm that might help with supporting patients in getting better and more targeted advice. This could potentially reduce unnecessary attendances at emergency departments and community walk-in centres. However, there is as yet no rigorous evidence available.

A key strength and application area of AI has been imaging and diagnostics, and this is something that could be put to good use during the pandemic. For example, a Chinese team trained a deep learning neural network to identify COVID-19 from chest X-rays and to distinguish this from other forms of pneumonia. ${ }^{13}$ If applied successfully in clinical practice, such an AI-supported approach could help protect healthcare staff and speed up the process of isolating and potentially tracking patients. However, care needs to be taken with results reported at this early stage. A review of 31 diagnostic and prediction models found that all of the models were at high risk of introducing bias and that the accuracy and performance estimates were likely to be overly optimistic. ${ }^{14}$ In order to speed up training of algorithms and to enhance their performance, shared data repositories should be built up globally, and the transparency of reporting needs to be enhanced.

Lastly, AI has the potential to support the treatment of COVID-19 through the development of new drugs and the redeployment of existing drugs. For example, large numbers of research papers accessible through the COVID-19 Open Research Database can be analysed quickly using machine learning to extract relevant knowledge about drugs that might be beneficial for the treatment of COVID-19. AI has also been used in the race for the development of vaccines and treatments. Hong Kong-based company Insilico Medicine reported that it had developed six new molecules that could potentially halt viral replication.

AI has potential to help health systems to fight COVID-19 through these initiatives around predicting and reducing spread, and by supporting diagnosis and treatment. There are open questions about data quality, transferability of results across settings and health systems, the performance of algorithms when actually used in clinical systems, and about access to data and protection of privacy. The crisis provides us with an opportunity to gain a glimpse of the future and to ponder these questions.

\section{DATA SHARING}

The rapid responses to COVID-19 have substantial implications for how healthcare data are used. Understandably, it has been a priority to make data quickly available to support disease surveillance and to inform operational requirements such as hospital capacity planning and resource management. There is also a broad range of urgent research needs, such as studies of virus mutations, patient risk factors, clinical outcomes and drug trials. ${ }^{15}$ Ultimately, the aim was to have data-driven public policy decisions on testing and tracing strategy, health system management, targeted isolation advice, social distancing rules and freedom of movement. Achieving these various objectives necessitates a rich collection of (usually pseudonymised) patient data, including demographics, prior conditions and medications, social circumstances, genome analysis, laboratory test results, diagnostic imaging and clinical narratives.

Analytics that address the whole picture will need to link data from multiple organisations and health record systems, posing challenges to enabling safe linkage while maintaining information security and managing the risks of reidentification. ${ }^{16}$ In the UK, an exceptional legal basis has been provided for this by the Secretary of State for Health and Social Care activating the Health Service (Control of Patient Information) Regulations, which requires affected organisations to "process confidential patient information... 
where the confidential patient information to be processed is required for a Covid-19 Purpose and will be processed solely for that COVID-19 Purpose' (italics added). ${ }^{17}$

How is this viewed by the general public? Surveys often find that sharing of health data by clinicians for legitimate care purposes is overwhelmingly trusted, but some studies have shown distrust in research use by pharmaceutical companies or academics even when data are anonymised. ${ }^{18}$ In the height of the pandemic, it is very likely that such cautions are mentally suspended by citizens, rather like the almost universal practice of hasty and uncritical acceptance of software licensing terms and conditions so that you can get on with using the product. However, with the 'genie out of the bottle', will governments, academics and industry be keen to return to the stricter regime of 'normal' information governance? Will there be a genuine citizen dialogue to see if there is a 'new normal' of easier data access? Or is there a risk of democratic nations semiconsciously drifting towards the kinds of citizen data exploitation seen in countries with repressive administrations? In an era of increasing concerns about the seemingly uncheckable powers of global tech companies, the rise of antiexpert right-wing populism and the anticipated economic catastrophe following the pandemic, these are serious issues that demand transparent ethical consideration.

\section{DISCUSSION}

The COVID-19 crisis has led to extraordinary rapid transformations of service delivery using telehealth technologies, thus showing that these technologies had already reached the level of maturity required to be deployed within healthcare systems at pace and scale: they were waiting in the wings. It is quite conceivable that digital consultations will remain the norm even when this pandemic is over. The pandemic has also led to rapid changes in the extent to which health data are being shared, both for direct care and for secondary purposes. It is less obvious, however, how the 'emergency' governance of health data would sustain after the crisis without causing a confrontation with public trust. For AI, finally, there appear to be numerous opportunities. However, few concrete achievements have been reported in the few months that we are now into the pandemic. It is yet unclear whether AI technologies have sufficient readiness to save the world when they are most needed, but no doubt, the near future will tell us more. Despite the pace of current changes, it is pertinent that we capture and share what can be learnt from these pandemic times. To be continued.

Funding NP's time was partly funded by the National Institute for Health Research (NIHR) Applied Research Collaboration Greater Manchester. The views expressed in this publication are those of the authors and not necessarily those of the NHS, the NIHR or the Department of Health and Social Care. The funder of the study had no role in study design, data collection, data analysis, data interpretation, or writing of the report.
Competing interests None declared.

Provenance and peer review Commissioned; internally peer reviewed.

Open access This is an open access article distributed in accordance with the Creative Commons Attribution Non Commercial (CC BY-NC 4.0) license, which permits others to distribute, remix, adapt, build upon this work non-commercially, and license their derivative works on different terms, provided the original work is properly cited, appropriate credit is given, any changes made indicated, and the use is non-commercial. See: http://creativecommons.org/licenses/by-nc/4.0/.

ORCID iD

Philip Scott http://orcid.org/0000-0002-6289-4260

\section{REFERENCES}

1 Lacktman NM, Acosta JN, Levine SJ. 50-State survey of telehealth commercial payer statutes. Foley.com/Telemedicine, 2019. Available: https://www.foley.com/-/media/files/insights/health-care-law-today/ 19mc21486-50state-survey-of-telehealth-commercial.pdf

2 HealthcarelTNews. The impact of COVID-19 on health tech adoption in the UK. Available: https://www.healthcareitnews.com/news/ europe/impact-covid-19-health-tech-adoption-uk

3 British Medical Association. COVID-19: steps for GP practices to take. Available: https://www.bma.org.uk/advice-and-support/covid19/practical-guidance/covid-19-steps-for-gp-practices-to-take

4 US Department of Health and Human Sciences. Notification of enforcement discretion for telehealth remote communications during the COVID-19 nationwide public health emergency. Available: https:// www.hhs.gov/hipaa/for-professionals/special-topics/emergencypreparedness/notification-enforcement-discretion-telehealth/index.html

5 Department of Health. Urgent written statement to the assembly by health minister Robin Swann - Thursday 19 March 2020 at 14.00. Health and social care planning for the period mid-March to mid-April during the delay phase for the coronavirus. Available: https://www.health-ni.gov.uk/sites/default/files/publications/health/ Coronavirus-19-03-2020.pdf

6 Greenhalgh T, Koh GCH, Car J. Covid-19: a remote assessment in primary care. BMJ 2020;368:m1182.

7 Turer RW, Jones I, Rosenbloom ST, et al. Electronic personal protective equipment: a strategy to protect emergency department providers in the age of COVID-19. J Am Med Inform Assoc 2020;27:967-71.

8 Hollander JE, Carr BG. Virtually perfect? Telemedicine for Covid-19. N Engl J Med 2020;382:1679-81.

9 Royal College of Physicians. COVID-19 and mitigating impact on health inequalities. Available: https://www.rcplondon.ac.uk/news/ covid-19-and-mitigating-impact-health-inequalities

10 Fraser H, Coiera E, Wong D. Safety of patient-facing digital symptom checkers. Lancet 2018;392:2263-4.

11 Joshi I, Morley J. Artifiical intelligence: how to get it right. Putting policy into practice for safe data-driven innovation in health and care. London: NHSX, 2019.

12 NHS England. Digital contact tracing: protecting the NHS and saving lives. Available: https://www.nhsx.nhs.uk/blogs/digital-contacttracing-protecting-nhs-and-saving-lives/

13 Li L, Qin L, Xu Z, et al. Artificial intelligence distinguishes covid-19 from community acquired pneumonia on chest CT. Radiology 2020:200905

14 Wynants L, Van Calster B, Bonten MMJ, et al. Prediction models for diagnosis and prognosis of covid-19 infection: systematic review and critical appraisal. BMJ 2020;369:m1328.

15 Health Data Research UK. Priority research questions. Available: https://www.hdruk.ac.uk/wp-content/uploads/2020/04/200414-HDRUK-priority-research-questions-002.pdf

16 Chowdury H. Microsoft's UK boss backs NHS data centre amid privacy concerns. Daily Telegraph, 2020.

17 UK Government. Coronavirus (COVID-19): notification to organisations to share information. Available: https://www.gov.uk/ government/publications/coronavirus-covid-19-notification-of-datacontrollers-to-share-information [Accessed 4 May 2020].

18 Patil S, Lu H, Saunders CL, et al. Public preferences for electronic health data storage, access, and sharing - evidence from a panEuropean survey. J Am Med Inform Assoc 2016;23:1096-106. 